\title{
Impaction of food material wrapped in a plastic sheet in a huge intraluminal duodenal diverticulum
}

\author{
Umid Kumar Shrestha $a^{\mathrm{a}, \mathrm{b}^{*}}$
}

a Manipal College of Medical Sciences \& Manipal Teaching Hospital, Pokhara, Nepal ${ }^{b}$ Kaski Sewa Hospital and Research Center, Pokhara, Nepal

\section{Accepted on}

November $15^{\text {th }}, 2012$

\section{DOI Name}

10.3126/jaim.v2i1.7631

\section{Keywords}

Endoscopy, intraluminal duodenal diverticulum, foreign body

\section{Citation}

Shrestha UK. Impaction of food material wrapped in a plastic sheet in a huge intraluminal duodenal diverticulum. Journal of Advances in Internal Medicine 2013;02(01):14-5.

\section{* Corresponding author}

Department of Medicine,

Manipal College of Medical Sciences \& Manipal Teaching Hospital, Pokhara, Nepal

Email address - umidshrestha@gmail.com

\section{CASE}

A 30-year-old male presented with a vague upper abdominal discomfort for 5 days. He complained of nausea and loss of appetite, but there was no vomiting. He was non-alcoholic and non-smoker. He was a businessman by occupation and had a small grocery store business. He had accidentally ingested some food material wrapped in a plastic sheet about 7 days prior to the presentation, but had not recovered the plastic sheet in the stool since then. His physical examination and laboratory investigation including serum amylase and liver function test were normal. The upper gastrointestinal (UGI) endoscopy was done, the finding of which is shown in the figure 1 . What is the diagnosis of the patient?

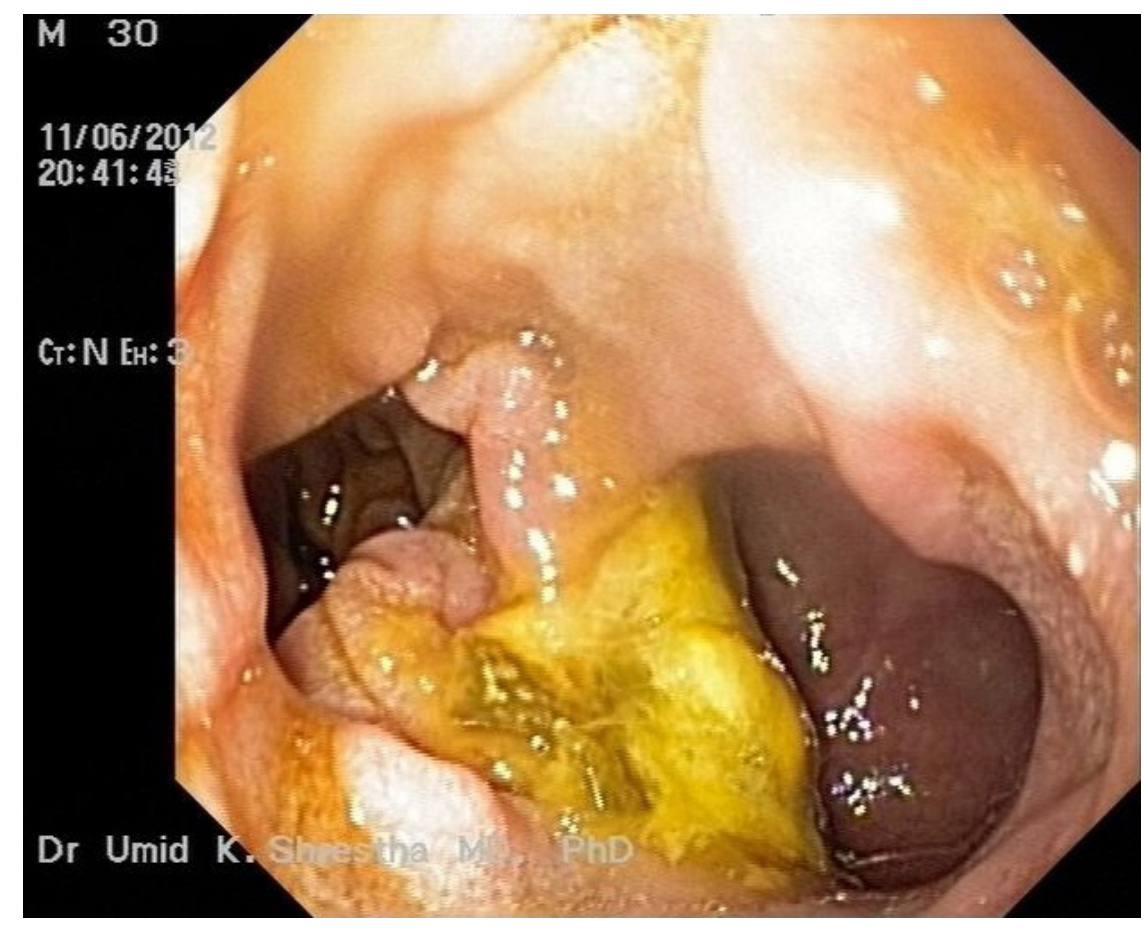

Figure 1: Intraluminal diverticulum in the second part of duodenum containing a food material wrapped in a plastic sheet

Answer: Intraluminal diverticulum in the second part of duodenum containing a food material wrapped in a plastic sheet

The UGI endoscopy done at the time of presentation demonstrated a huge intraluminal diverticulum in the second part of duodenum. The plastic sheet containing the food particles was lodged in the duodenal diverticulum. The plastic sheet (along with residual food particles) was retrieved endoscopically after a seventh day of ingestion. The figure 2 shows the UGI endoscopy image after 
the endoscopic retrieval of plastic sheet.

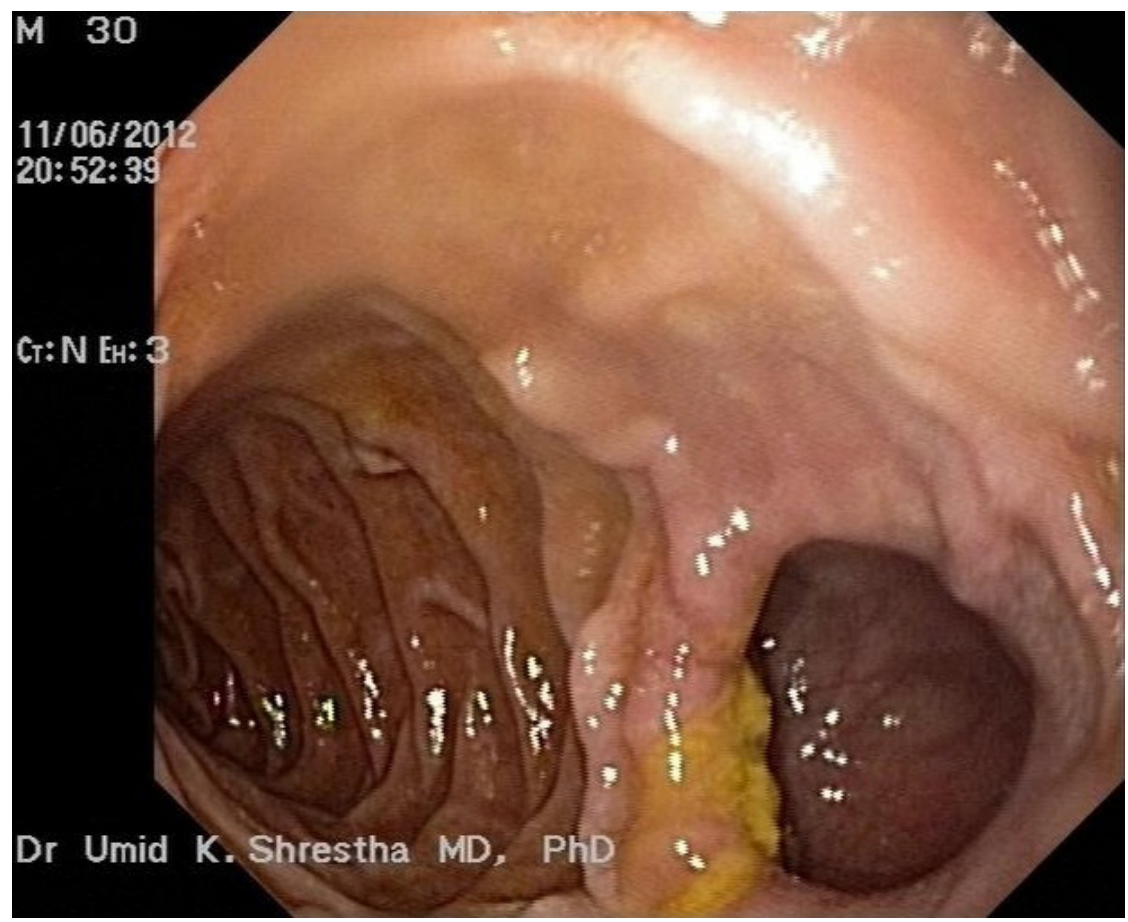

Figure 2: Intraluminal duodenal diverticulum after the endoscopic removal of a foreign body (food material wrapped in a plastic sheet)

There has been one report of ingestion of plastic sheeting with a subsequent occurrence of colonic perforation. ${ }^{1}$ However, there has been no report of the retention of the plastic sheet in the duodenal diverticulum. Duodenal duplication is rare and represents only $4-12 \%$ of duplications of the alimentary tract. ${ }^{2}$ Intraluminal duodenal diverticulum (also called windsock diverticula) is a rare form of duodenal duplication and may be asymptomatic for years. Some patients may present with recurrent pancreatitis or biliary problems; however, the most common symptoms are those of incomplete duodenal obstruction. The obstruction may be precipitated by retention of vegetable material or foreign bodies. One report has mentioned about the retention of two marbles in an intraluminal duodenal diverticulum in a 41-year-old man, the marbles being swallowed during childhood. ${ }^{3}$ In conclusion, intraluminal duodenal diverticulum is a rare disorder in which a foreign body can often be lodged. If an ingested foreign body is not excreted in the stool, the possible retention in the intestinal tract should always be considered and a thorough investigation is warranted.

\section{REFERENCES}

1) Fernando GC. Colonic perforation following ingestion of plastic sheeting. Med Sci Law 1989;29:263-4.

2) Moreno P, Lazo MD, Andrade RJ, et al. Unusual Duodenal Duplication Cyst Associated with Partial Gastric Diverticulum in a Middle-Aged Woman. Are they Congenital or Acquired? Dig Dis Sci 2002;47:304-8.

3) Abdel-Hafiz AA, Birkett Dh, Ahmed MS. Congenital duodenal diverticula: A report of three cases and a review of the literature. Surgery 1988; 104:74-8. 